\title{
Genetic variants of methionine metabolism and X-ALD phenotype generation: results of a new study sample
}

\author{
Semmler, A ; Bao, X ; Cao, G ; Köhler, W ; Weller, M ; Aubourg, P ; Linnebank, M
}

\begin{abstract}
X-linked adrenoleukodystrophy (X-ALD) is the most common inherited leukodystrophy. Nevertheless, no genotype-phenotype correlation has been established so far. Unidentified modifier genes or other cofactors are suspected to modulate phenotype and prognosis. We recently described polymorphisms of methionine metabolism as possible disease modifiers in X-ALD. To retest these findings, we analyzed 172 new DNA samples of X-ALD patients from different populations (France, Germany, USA, China) by genotyping eight genetic variants of methionine metabolism, including DHFR c.594+59del19bp, CBS c. $844_{8} 55$ ins 68, MTRc.2756A > G, MTHFRc.677C > Tandc. $1298 A>C, M T R R c .60 A>G, R F C 1 c .80 G>$ A, andTc $2 c .776 C>G . W$ ecomparedthreeX-ALDphenotypes : childhood-onsetcerebraldemyelinatinginflammatoryty 82), adulthoodonsetwith focalcerebraldemyelination $(A C A L D ; n=38)$, andadulthoodonsetwithoutcerebraldemyelinatio 52).Theassociationof genotypesandphenotypeswasanalyzedwithunivariatetwo-sidedPear son'schi(2).Inthecomparisor Gwasassociatedwith $X-A L D$ phenotypes $(\operatorname{chi}(2)=6.1 ; P=0.048)$.TheprevalenceoftheGGgenotypeofTc 2 c. $776 C>$ GwashigherinpatientswithCNSdemyelinationcomparedtothosewithoutCNSdemyelination $(\operatorname{chi}(2)=4.42 ; P=$ 0.036).TheGGgenotypewasalsomore frequentinCCALDcomparedtoAMN $(\operatorname{chi}(2)=4.7 ; P=0.031)$. Theotherpolymorp ALDphenotypegeneration.
\end{abstract}

DOI: https://doi.org/10.1007/s00415-009-5114-6

Posted at the Zurich Open Repository and Archive, University of Zurich

ZORA URL: https://doi.org/10.5167/uzh-21177

Journal Article

Accepted Version

Originally published at:

Semmler, A; Bao, X; Cao, G; Köhler, W; Weller, M; Aubourg, P; Linnebank, M (2009). Genetic variants of methionine metabolism and X-ALD phenotype generation: results of a new study sample. Journal of Neurology, 256(8):1277-1280.

DOI: https://doi.org/10.1007/s00415-009-5114-6 
Genetic variants of methionine metabolism and X-ALD phenotype generation: results of a new study sample

Alexander Semmler ${ }^{1 *}$, Xinhua Bao ${ }^{2 *}$, Guangna $\mathrm{Cao}^{2}$, Wolfgang Köhler ${ }^{3}$, Michael Weller ${ }^{1}$, Patrick Aubourg ${ }^{4}$, Michael Linnebank ${ }^{1}$

${ }^{1}$ University Hospital Zürich, Department of Neurology, Zürich, Switzerland

${ }^{2}$ Peking University, Department of Pediatrics, First Hospital, Peking, China

${ }^{3}$ Sächsisches Krankenhaus Hubertusburg, Department of Neurology, Wermsdorf, Germany

${ }^{4}$ INSERM U745, Department of Pediatric Neurology, Hôpital Saint-Vincent de Paul,

University René Descartes, Paris, France

*A. S. and X.B. These authors contributed equally to this work.

Correspondence: Dr. med. Michael Linnebank, University of Zürich, Department of Neurology, Frauenklinikstrasse 26, 8091 Zürich, fon: (41) 44 2555544, fax: (41) 442554507 , E-Mail: michael.linnebank@,usz.ch

Running title: Methionine metabolism and X-ALD

Word count text: 1298

Word count abstract: 241

Character count title: 89 


\section{Abstract}

X-linked adrenoleukodystrophy (X-ALD) is the most common inherited leukodystrophy. Nevertheless no genotype/phenotype correlation has been established yet. Unidentified modifier genes or other co-factors are suspected to modulate phenotype and prognosis. We recently described polymorphisms of methionine metabolism as possible disease modifiers in X-ALD.

To retest these findings, we analyzed 172 new DNA- samples of X-ALD patients from different populations (France, Germany, USA, China) by genotyping the eight genetic variants of methionine metabolism including DHFR c.594+59del19bp, CBS c.844_855ins68, MTR c. $2756 \mathrm{~A}>\mathrm{G}$, MTHFR c. $677 \mathrm{C}>$ T and c. $1298 \mathrm{~A}>\mathrm{C}$, MTRR c. $60 \mathrm{~A}>\mathrm{G}, \mathrm{RFC} 1 \mathrm{c} .80 \mathrm{G}>\mathrm{A}$, and Tc2 c.776C $>$ G. We compared the three X-ALD phenotypes childhood onset cerebral demyelinating inflammatory type (CCALD; $n=82$ ), adulthood onset with focal cerebral demyelination (ACALD; $\mathrm{n}=38$ ), and adulthood onset without cerebral demyelination (AMN; $\mathrm{n}=52$ ). Association of genotypes and phenotypes was analyzed with univariate two sided Pearson`s $\chi^{2}$.

The G-allele of Tc2 c.776C $>\mathrm{G}$ was associated with X-ALD phenotypes in the comparison between $\operatorname{AMN}$ and CCALD $\left(\chi^{2}=6.1 ; p=0.048\right)$. The prevalence of the GG- genotype of Tc2 c.776C $>\mathrm{G}$ was higher in patients with $\mathrm{CNS}$ demyelination compared to those without CNSdemyelination $\left(\chi^{2}=4.42 ; p=0.036\right)$. The GG- genotype was more also more frequent in CCALD compared to $\operatorname{AMN}\left(\chi^{2}=4.7 ; \mathrm{p}=0.031\right)$. The other polymorphisms did not reveal significant associations in this study sample.

Whereas any influence of other polymorphisms of methionine metabolism was not confirmed, the present study supports the previously made observation that the Tc2 genotype contributes to X-ALD phenotype generation. 
Search terms: [95] Association studies in genetics; [155] Leukodystrophies; [159]

Peroxisomes; [153] Metabolic disease (inherited) 


\section{Introduction}

X-linked adrenoleukodystrophy (X-ALD, OMIM \#300100) is caused by defects of the ABCD1 gene on chromosome Xq28 resulting in an impairment of peroxisomal beta-oxidation and the accumulation of saturated very long chain fatty acids (VLCFA). The minimum frequency of hemizygotes plus heterozygotes is 1:16,800 in the United States, suggesting that $\mathrm{X}-\mathrm{ALD}$ is the most common inherited leukodystrophy [3].

$\mathrm{X}$-ALD is characterized by a highly variable clinical spectrum. It ranges from cerebral demyelinating, inflammatory childhood phenotypes associated with a poor prognosis (CCALD), over milder adulthood disease with focal demyelination in the central nervous system (ACALD), to adulthood forms without central nervous system demyelination and considerably slower symptom progression (AMN). The appearance of cerebral demyelination is crucial for the phenotype, prognosis and therapy of X-ALD. The development of the phenotype can not be predicted by biochemical factors like levels of VLCFA. In addition, no genotype/phenotype correlation has been established [13]. Even within closely related patients the clinical course may range widely. Thus, yet unidentified modifier genes or other cofactors are likely to modulate the phenotypic variation and disease severity [5-8].

One possible X-ALD disease modifier might be methionine metabolism. Methionine metabolism plays a crucial role in DNA methylation and in providing methyl groups necessary for building brain myelination. It is also involved in the generation of glutathione that is necessary for defense against oxidative stress and in the regulation of inflammatory processes [15]. The metabolism is of marked inter-individual variety due to the existence of functionally relevant polymorphisms and differences in vitamin availability, which are important cofactors in methionine metabolism (vitamin B12, B6, folic acid). We have previously reported that polymorphisms of methionine metabolism are associated with the clinical phenotype of X-ALD. The insertion allele of cystathionine beta-synthase (CBS) c.844_845ins68 was significantly more common in AMN patients than in the cerebral 
demyelinating forms CCALD and ACALD in a sample of 86 Dutch and German patients [12]. In addition to that, the GG genotype of transcobalamin 2 (Tc2) c.776C $>\mathrm{G}$ was more common in ACALD than in AMN, and a combined risk genotype was more common in ACALD than in AMN. The combined risk genotype was defined as: presence of at least one of the genotypes transcobalamin 2 (Tc2) c. $776 \mathrm{C}>\mathrm{G}(\mathrm{GG})$, methylenetetrahydrofolate reductase (MTHFR) c.677C $>$ T $(\mathrm{TT})$ or methionine synthase $(\mathrm{MTR})$ c.2756A $>\mathrm{G}(\mathrm{AG} / \mathrm{GG})$ [9]. Here we studied the genotypes of 172 additional X-ALD patients to retest our previous data.

\section{Materials and Methods}

\section{$\underline{\text { Patients }}$}

We analyzed 172 DNA samples: 34 DNA samples from China (CCALD: $n=21$; ACALD: n=9; AMN: $n=4), 110$ samples from France (CCALD: $n=50$; ACALD: $n=25 ;$ AMN: $n=35), 8$ DNA samples from Germany (ACALD: $n=4 ; A M N$ : $n=4$ ) and 20 samples from the USA (CCALD: $n=11 ;$ AMN: n=9). Patients from China were of Han ethnicity, patients from France, Germany and USA of Caucasian origin. All patients or their trustees have given written informed consent. The study was approved by the local ethical committees.

\section{Genotyping}

Genomic DNA prepared from peripheral leukocytes was used for genotyping by PCR amplification and restriction analysis of eight genetic variants of methionine metabolism including the intronic deletion dihydrofolate reductase (DHFR) c.594+59del19bp (affecting the transcript level; GenBank NM_000791.3), the splice alteration cystathionine beta-synthase (CBS) c.844_855ins68 (affecting the transcript level; GenBank S78267.1), and the missense mutations (i.e., leading to amino acid exchanges) methionine synthase (MTR) c.2756A $>$ G (p.D919G; rs1805087), methylenetetrahydrofolate reductase (MTHFR) c.677C > T (p.A222V; 
rs 1801133) and c.1298A>C (p.E429A; rs1801131), methionine synthase reductase MTRR c.60A $>\mathrm{G}$, reduced folate carrier $1(\mathrm{RFC} 1) \mathrm{c} .80 \mathrm{G}>\mathrm{A}(\mathrm{p} . \mathrm{R} 27 \mathrm{H} ;$ rs 1051266$)$, and transcobalamin $2(\mathrm{Tc} 2)$ c.776C $>\mathrm{G}$ (p.P259R; rs1801198).[10]

\section{$\underline{\text { Statistics }}$}

The association of genotypes and phenotypes was analyzed with univariate two sided Pearson`s $\chi^{2}$ tests. As this is a confirmatory study, we did not correct for multiple testing.

\section{Results}

Analyses with two degrees of freedom (all genotypes) revealed an association of the G-allele of $\mathrm{Tc} 2 \mathrm{c} .776 \mathrm{C}>\mathrm{G}$ with the $\mathrm{X}-\mathrm{ALD}$ phenotype in the comparison between AMN and CCALD $\left(\chi^{2}=6.09 ; \mathrm{p}=0.048\right)$, but not in the comparison between $\mathrm{AMN}$ and the pooled demyelinating phenotypes CCALD+ACALD, $\chi^{2}=4.74 ; p=0.093$. The other polymorphisms did not reveal significant associations in this study sample (table 1). If only the Caucasian patients without the patients from China or subgroups divided by populations were analyzed, the differences of the frequencies of the Tc2 genotype in different X-ALD phenotypes were not significant (not shown). The prevalence of the GG-genotype of Tc2 c.776C $>\mathrm{G}$ was higher in patients with CNS demyelination than in those without CNS-demyelination $\left(\chi^{2}=4.42 ; p=0.036\right)$. Additionally, the GG- genotype was more frequent in patients with the most severe phenotype, CCALD, than in patients with $\operatorname{AMN}\left(\chi^{2}=4.71 ; p=0.031\right)$ (table 2$)$.

\section{Discussion}

In the present study consisting of 172 patients with CCALD, ACALD and AMN we found that the G-allele of the Tc2 c.776C $>$ G polymorphism is associated with the occurrence of CNS demyelination in X-ALD patients. The G-allele of Tc2 c.776C $>$ G leads to the amino acid substitution p.P259R that seems to affect the affinity of the transcobalamin 2 protein to 
vitamin B12 and the ability to transport vitamin B12 into tissues $[1,2,4]$. Vitamin B12 is necessary to synthesize S-adenosylmethionine, which serves as methyl group donor for CNS myelination. Accordingly, vitamin B12 deficiency can lead to demyelination of the central nervous system in otherwise healthy individuals [16].

Methotrexate is a widely used chemotherapeutic drug, which may lead to neurotoxic sideeffects accompanied by cerebral white matter changes. Methotrexate reduces the vitamin B12-dependent synthesis of S-adenosylmethionine in the central nervous system [14]. In a series of 68 patients treated with methotrexate the G-allele and the GG-genotype of Tc2 c.776C $>\mathrm{G}$ were associated with the occurrence of white matter changes of the brain [11]. We conclude that it is unlikely that the GG-genotype of $\mathrm{Tc} 2 \mathrm{c} .776 \mathrm{C}>\mathrm{G}$ is the main determinant of the different X-ALD phenotypes. This observation may serve as an indicator of the role of the methionine metabolism in X-ALD disease progression in general. We speculate that the influence of the GG-genotype of $\mathrm{Tc} 2 \mathrm{c} .776 \mathrm{C}>\mathrm{G}$ on functional availability of vitamin $\mathrm{B} 12$ and of SAM synthesis may dispose X-ALD patients to demyelination of the central nervous system.

In a previous study with a sample of 86 Dutch and German patients, we observed an association of X-ALD phenotypes with additional genetic variants of the methionine metabolism $[9,12]$. In the actual sample we only confirmed the association of the G-allele of the polymorphism $\mathrm{Tc} 2 \mathrm{c} .776 \mathrm{C}>\mathrm{G}$ with the occurrence of $\mathrm{CNS}$ demyelination in X-ALD patients, whereas the other previous observations were not confirmed. We can not completely exclude differences between the previous and the actual patient samples that might have contributed to the differences in the results. Dietary influences concerning the uptake of amino acids or vitamins may speculatively modify effects of the analyzed polymorphisms on X-ALD phenotype generation, and dietary conditions might have differed between the old and 
the new patient sample. However, we conclude that only the influence of the Tc2 polymorphism is validated regarding the results of the present study, whereas the other previously reported findings have to be assumed as false positive as long as they are not confirmed in an additional X-ALD patient sample.

These data are limited as the association is of weak significance. If corrected for multiple testing or if analyzed in subgroups divided by populations or ethnicity, the results were not significant. However, this study was of confirmative character, and as the results concerning the Tc2 variant confirmed our previous observations in an independent sample of X-ALD patients, we consider the influence of the $\mathrm{Tc} 2 \mathrm{c} .776 \mathrm{C}>\mathrm{G}$ variant on phenotype generation in X-ALD as confirmed.

It seems warranted to examine the effect of experimentally induced vitamin B12, folate and SAM depletion in addition to vitamin B12, folate and SAM substitution on the phenotype of X-ALD mouse models to estimate whether clinical studies investigating the effect of vitamin or SAM substitution on the clinical course of X-ALD are justified.

\section{$\underline{\text { Acknowledgements }}$}

The USA samples were provided by Hugo and Ann Moser, Kennedy Krieger Institute. Grant: HD 39276 (NICHD). This study was supported by the European Leukodystrophies Association (M.L.). 
Table 1: All analysed polymorphisms and X-ALD phenotypes

\begin{tabular}{|c|c|c|c|c|c|}
\hline $\begin{array}{l}\text { Tc2 } \\
\text { c. } 776 \mathrm{C}>\mathrm{G}\end{array}$ & $\mathrm{CC}$ & $\mathrm{CG}$ & GG & CCALD vs. AMN & $\begin{array}{l}\text { CCALD+ACALD } \\
\text { vs. AMN }\end{array}$ \\
\hline CCALD & 0.19 & 0.42 & 0.39 & $\mathrm{p}=0.048$ & $\mathrm{p}=0.093$ \\
\hline ACALD & 0.34 & 0.32 & 0.34 & & \\
\hline AMN & 0.35 & 0.44 & 0.21 & & \\
\hline $\begin{array}{l}\text { MTR } \\
\text { c. } 2756 \mathrm{~A}>\mathrm{G}\end{array}$ & AA & $\mathrm{AG}$ & GG & CCALD vs. AMN & $\begin{array}{l}\text { CCALD/ACALD } \\
\text { vs. AMN }\end{array}$ \\
\hline CCALD & 0.72 & 0.25 & 0.03 & $p=0.364$ & $\mathrm{p}=0.393$ \\
\hline ACALD & 0.78 & 0.22 & 0 & & \\
\hline $\mathrm{AMN}$ & 0.69 & 0.31 & 0 & & \\
\hline $\begin{array}{l}\text { MTHFR } \\
\text { c. } 677 \mathrm{C}>\mathrm{T}\end{array}$ & $\mathrm{CC}$ & $\mathrm{CT}$ & TT & CCALD vs. AMN & $\begin{array}{l}\text { CCALD+ACALD } \\
\text { vs. AMN }\end{array}$ \\
\hline CCALD & 0.42 & 0.39 & 0.19 & $\mathrm{p}=0.940$ & $\mathrm{p}=0.970$ \\
\hline ACALD & 0.31 & 0.53 & 0.16 & & \\
\hline $\mathrm{AMN}$ & 0.40 & 0.42 & 0.18 & & \\
\hline $\begin{array}{l}\text { MTHFR } \\
\text { c. } 1298 \mathrm{~A}>\mathrm{C}\end{array}$ & AA & $\mathrm{AC}$ & $\mathrm{CC}$ & CCALD vs. AMN & $\begin{array}{l}\text { CCALD+ACALD } \\
\text { vs. AMN }\end{array}$ \\
\hline CCALD & 0.59 & 0.33 & 0.08 & $\mathrm{p}=0.721$ & $\mathrm{p}=0.964$ \\
\hline ACALD & 0.42 & 0.53 & 0.05 & & \\
\hline $\mathrm{AMN}$ & 0.51 & 0.41 & 0.08 & & \\
\hline $\begin{array}{l}\text { CBS } \\
\text { c. } 844 \_845 \text { ins } 68\end{array}$ & $\mathrm{dd}$ & di & ii & CCALD vs. AMN & $\begin{array}{l}\text { CCALD+ACALD } \\
\text { vs. AMN }\end{array}$ \\
\hline CCAL̄D & 0.89 & 0.11 & 0 & $\mathrm{p}=0.659$ & $\mathrm{p}=0.989$ \\
\hline ACALD & 0.89 & 0.11 & 0 & & \\
\hline AMN & 0.89 & 0.11 & 0 & & \\
\hline $\begin{array}{l}\text { MTRR } \\
\text { c. } 60 \mathrm{~A}>\mathrm{G}\end{array}$ & AA & $\mathrm{AG}$ & GG & CCALD vs. AMN & $\begin{array}{l}\text { CCALD+ACALD } \\
\text { vs. AMN }\end{array}$ \\
\hline CCALD & 0.14 & 0.42 & 0.44 & $\mathrm{p}=0.226$ & $\mathrm{p}=0.502$ \\
\hline ACALD & 0.24 & 0.54 & 0.22 & & \\
\hline $\mathrm{AMN}$ & 0.15 & 0.57 & 0.28 & & \\
\hline $\begin{array}{l}\text { RFC1 } \\
\text { c. } 80 \mathrm{G}>\mathrm{A}\end{array}$ & GG & $\mathrm{AG}$ & AA & CCALD vs. AMN & $\begin{array}{l}\text { CCALD+ACALD } \\
\text { vs. AMN }\end{array}$ \\
\hline CCALD & 0.31 & 0.47 & 0.22 & $\mathrm{p}=0.948$ & $\mathrm{p}=0.822$ \\
\hline ACALD & 0.27 & 0.43 & 0.30 & & \\
\hline $\mathrm{AMN}$ & 0.33 & 0.46 & 0.21 & & \\
\hline $\begin{array}{l}\text { DHFR } \\
\text { c.594+59del19b }\end{array}$ & dd & di & ii & CCALD vs. AMN & $\begin{array}{l}\text { CCALD+ACALD } \\
\text { vs. AMN }\end{array}$ \\
\hline $\begin{array}{l}\mathrm{p} \\
\text { CCALD }\end{array}$ & 0.14 & 0.51 & 0.35 & $\mathrm{p}=0.253$ & $\mathrm{p}=0.238$ \\
\hline
\end{tabular}




$\begin{array}{llll}\text { ACALD } & 0.12 & 0.54 & 0.34 \\ \text { AMN } & 0.06 & 0.48 & 0.46\end{array}$

Table 1: Result of all polymorphisms analysed and X-ALD phenotypes CCALD $(n=82)$, $\operatorname{ACALD}(\mathrm{n}=38)$, and $\operatorname{AMN}(\mathrm{n}=52)$.

Table 2: Tc2 c.776-GG genotypes and X-ALD phenotypes

$\begin{array}{lllll}\text { Tc2 } & \mathrm{CC} / & \mathrm{GG} & \text { pure AMN vs. } & \text { pure AMN vs. } \\ \text { c.776C }>\mathrm{G} & \mathrm{CG} & & \text { CCALD } & \text { CCALD+ALMN } \\ \text { CCALD } & 0.61 & 0.39 & \mathrm{p}=0.031 & \mathrm{p}=0.036 \\ \text { ACALD } & 0.66 & 0.34 & & \\ \text { AMN } & 0.79 & 0.21 & & \end{array}$

Table 2: Tc2 c.776-GG genotype and X-ALD phenotypes CCALD ( $\mathrm{n}=82)$, ACALD $(\mathrm{n}=38)$, and $\operatorname{AMN}(\mathrm{n}=52)$. 


\section{References}

1. Afman LA, Van Der Put NM, Thomas CM, Trijbels JM, Blom HJ (2001) Reduced vitamin B12 binding by transcobalamin II increases the risk of neural tube defects. Qjm 94:159-166

2. Alessio AC, Hoehr NF, Siqueira LH, Bydlowski SP, Annichino-Bizzacchi JM (2007) Polymorphism C776G in the transcobalamin II gene and homocysteine, folate and vitamin B12 concentrations. Association with MTHFR C677T and A1298C and MTRR A66G polymorphisms in healthy children. Thromb Res 119:571-577

3. Bezman L, Moser AB, Raymond GV, Rinaldo P, Watkins PA, Smith KD, Kass NE, Moser HW (2001) Adrenoleukodystrophy: incidence, new mutation rate, and results of extended family screening. AnnNeurol 49:512-517

4. Castel-Dunwoody KM, Kauwell GP, Shelnutt KP, Vaughn JD, Griffin ER, Maneval DR, Theriaque DW, Bailey LB (2005) Transcobalamin 776C->G polymorphism negatively affects vitamin B-12 metabolism. AmJClinNutr 81:1436-1441

5. Dodd A, Rowland SA, Hawkes SL, Kennedy MA, Love DR (1997) Mutations in the adrenoleukodystrophy gene. HumMutat 9:500-511

6. Gartner J, Braun A, Holzinger A, Roerig P, Lenard HG, Roscher AA (1998) Clinical and genetic aspects of X-linked adrenoleukodystrophy. Neuropediatrics 29:3-13

7. Heinzer AK, McGuinness MC, Lu JF, Stine OC, Wei H, Van der Vlies M, Dong GX, Powers J, Watkins PA, Smith KD (2003) Mouse models and genetic modifiers in Xlinked adrenoleukodystrophy. Adv Exp Med Biol 544:75-93 
8. Kok F, Neumann S, Sarde CO, Zheng S, Wu KH, Wei HM, Bergin J, Watkins PA, Gould S, Sack G (1995) Mutational analysis of patients with X-linked adrenoleukodystrophy. HumMutat 6:104-115

9. Linnebank M, Kemp S, Wanders RJ, Kleijer WJ, van der Sterre ML, Gartner J, Fliessbach K, Semmler A, Sokolowski P, Kohler W, Schlegel U, Schmidt S, Klockgether T, Wullner U (2006) Methionine metabolism and phenotypic variability in X-linked adrenoleukodystrophy. Neurology 66:442-443

10. Linnebank M, Moskau S, Farmand S, Fliessbach K, Kolsch H, Bos M, Grothe C, Becker D, Harbrecht U, Pohl C, Wullner U, Klockgether T (2006) Homocysteine and Carotid Intima-Media Thickness in a German Population. Lack of Clinical Relevance. Stroke

11. Linnebank M, Moskau S, Jurgens A, Simon M, Semmler A, Orlopp K, Glasmacher A, Bargart C, Vogt-Schaden M, Urbach H, Schmidt-Wolf IG, Pels H, Schlegel U (2008) Association of genetic variants of methionine metabolism with MTX-induced CNS white matter changes in patients with primary central nervous system lymphoma. Neuro Oncol

12. Linnebank M, Semmler A, Kleijer WJ, van der Sterre ML, Gartner J, Fliessbach K, Sokolowski P, Kohler W, Schlegel U, Klockgether T, Wanders RJ, Schmidt S, Wullner U, Kemp S (2006) The cystathionine beta-synthase variant c.844_845ins68 protects against CNS demyelination in X-linked adrenoleukodystrophy. HumMutat 27:1063-1064

13. Moser HW, Mahmood A, Raymond GV (2007) X-linked adrenoleukodystrophy. Nat Clin Pract Neurol 3:140-151 
14. Surtees R, Clelland J, Hann I (1998) Demyelination and single-carbon transfer pathway metabolites during the treatment of acute lymphoblastic leukemia: CSF studies. JClinOncol 16:1505-1511

15. Tchantchou F (2006) Homocysteine metabolism and various consequences of folate deficiency. J Alzheimers Dis 9:421-427

16. Toohey JI (2006) Vitamin B12 and methionine synthesis: a critical review. Is nature's most beautiful cofactor misunderstood? Biofactors 26:45-57 